\title{
Sistema, autopoiesis y entropía en los desafíos educativos contemporáneos*
}

\author{
José Luis Arriaga Ornelas ${ }^{1}$ \\ (D) http://orcid.org/0000-0002-0498-8461 \\ Universidad Autónoma del Estado de México
}

DOI: http://dx.doi.org/ 10.17081/eduhum.19.33.2644

Recibido: 20 de mayo de 2016

Aceptado: 30 de enero de 2017

\section{Systems, autopoiesis y entropy in contemporary educational challenges}

Palabras clave:

Sistemas, Clausura operativa, Autopoiesis, Información, Entropía.

Key words:

Systems, Operational closure, Autopoiesis, Information, Entropy.

\begin{abstract}
Resumen
El presente es un texto de revisión de tres principios teóricos cuyo valor heurístico no sólo alcanza para explicar un fenómeno tan importante como es la Educación, sino para aventurarse en la reflexión sobre los destinatarios de las prácticas educativas: los estudiantes. Utilizando planteamientos teóricos de Niklas Luhmann, Edgar Morin y Emilio Roger Ciurana, este trabajo ofrece el planteamiento hipotético de que los estudiantes corren el riesgo de caer en un proceso de degradación del orden informacional, en la medida en que no incrementan complejidad mental para gestionar los retos espistemológicos y societales que ofrece nuestro tiempo.
\end{abstract}

\begin{abstract}
The present article is a study for the review of three theoretical principles whose heuristic value is enough not only for explaining the most important phenomena of education, but also for getting into a reflection about educational practices on the final recipients: which are the students. Utilizing the basic theories of Niklas Luhmann, Edgar Morin y Emilio Roger Ciurana, this work offers a hypothetical argument that states that students run the risk of falling into a process of degradation of an informational order, in as much as they cannot cope with the mental complex capacity required to manage the epistemic challenges set forth by today's society.
\end{abstract}

Referencia de este artículo (APA): Arriaga, J. (2017). Sistema, autopoiesis y entropía en los desafíos educativos contemporáneos. En Revista Educación y Humanismo, 19(33), 271-288. http://dx.doi.org/10.17081/eduhum.19.33.2644

\footnotetext{
* Artículo de reflexión, derivado de la investigación "Innovación curricular y mentalidad del universitario", financiada por la Universidad Autónoma del Estado de México.

1. Doctor de las Ciencias Sociales de la Universidad Autónoma del Estado de México. jlarriagao@gmail.com
} 


\section{Introducción}

El tema de "educación y complejidad" encierra una paradoja, debido a que todo proceso de enseñanza es instructivo (Contreras, 1994), es decir, parte de una concepción de lo que es la persona humana o de lo que debería ser, y esta situación evidencia una complejidad reducida; pero pensar de modo complejo es una apuesta por la no reducción (Morin, 2005). En este texto se desea explorar la manera en que esa paradoja puede ser puesta en movimiento, presentándola como una "compuerta evolutiva" (Najmanovich, 2008) en el problema de la convivencia humana.

En su desarrollo, se desplegarán argumentos en el sentido de que el proceso educativo institucionalizado, que concibe a los individuos como sistemas psíquicos, contribuye a realizar lo que en la Teoría de Sistemas se nombra como "clausura operativa", en tanto que el pensamiento complejo representa la posibilidad de que esos sistemas no se degraden a sí mismos al mantenerse en un frágil equilibrio precisamente por la clausura que padecen, sino que se reorganicen hacia una apertura.

Por tratarse de un problema epistemológico, este texto busca llamar la atención sobre las maneras de pensar (mismas que no pueden separarse de la acción, pues, como dice Maturana Todo hacer es conocer). Para tal efecto, se decidió asumir las metáforas de "sistema", "autopoiesis" y “entropía”. En este sentido, los planteamientos que se realizarán se basan en dos premisas: la primera abreva de los planteamientos de la
Teoría de los Sistemas Sociales de Luhmann (1998) y percibe las prácticas educativas institucionalizadas en la sociedad contemporánea como recursivas, ya que se suceden unas a otras y autoreproducen el sistema Educación; la segunda recupera el planteamiento de Morin (2001) y Ciurana \& Regalado (2016), en el sentido de que solo es posible reducir la realidad fenoménica aumentando complejidad intelectual.

Para conseguir su objetivo, el trabajo se divide en tres partes: la primera concibe a la sociedad como un tipo de sistema emergente, auto-organizado y auto-referente (un todo cuyas propiedades no se pueden reducir a las de las unidades más pequeñas que lo componen, sino que surgen de las relaciones entre las partes) y a la educación como un sistema parcial de la sociedad contemporánea, que se apoya en las tesis de Luhmann. La segunda parte desarrolla la idea de Morin según la cual los sistemas vivientes dependen de una alimentación exterior, no solo material-energética, sino también organizacional-informacional. Y la tercera parte, en coincidencia con Ciurana, propone una manera de modificar tanto el estatuto epistemológico para comprender la diversidad humana, como la actitud del yo societal que tiene contacto con lo distinto, sin saber cómo hacerlo para no llegar a la censura y la confrontación. En términos generales, la propuesta se encamina a fomentar la convivencia social dialógica desde la epistemología del constructivismo (Von Foerster, 1998). 
El sistema educación y las máquinas no triviales

No es pertinente hablar de la educación en abstracto, conviene más referirse a prácticas educativas; es decir, a actos humanos intencionales que se presentan como una realidad fenoménica. Por tanto, la educación de la que se hablará aquí es la que hoy se asigna como responsabilidad de las instituciones educativas: la actividad sistemática e intencionada consistente en la transmisión de saber entre los miembros de una sociedad, hasta niveles en donde "la socialización no es suficiente para asegurar la capacidad del comportamiento adecuado" (Corsi y Esposito, \& Baraldi, 2006, p.96). Este tipo de prácticas educativas son contingentes, lo cual significa que sus contenidos y formas siempre se pueden concretar de otra(s) manera(s), así que no pueden calificarse de inevitables o necesarias.

Desde una racionalidad sistémica, que no presupone la existencia de estructuras, se puede hablar de esas prácticas educativas como formas de resolver un problema de la praxis cotidiana (el de instruir, socializar, evaluar a los integrantes de la sociedad), que "a su vez generan las condiciones que reproducen los problemas que han de resolver" (Mascareño, 2016, p.31). Lo que emerge de ese impulso por resolver el problema cotidiano de enseñar a los integrantes de la sociedad "lo que deben saber", generará así nuevas necesidades de enseñanza: enseñar a los que enseñarán, espacios para enseñar, formas de evaluar lo enseñado, etc. El modo en que todo este conjunto de acciones llega a estabili- zarse es por la característica autopoiética de un sistema, que consiste en operar como una red cerrada de autoproducción de los componentes que lo constituyen, basada en la recursividad y la clausura operativa. Esto último porque sus componentes se producen al interior del sistema y también estos lo producen. Entonces, pensar en prácticas educativas nos provee un sistema empíricamente observable, operativamente cerrado y autorreferencial.

La metáfora de "sistema" es muy rica, porque -dice Morin-, de una u otra manera, toda realidad conocida (desde el átomo hasta la galaxia, pasando por la molécula, la celula, el organismo y la sociedad puede ser concebida como un sistema, es decir, "como asociación combinatoria de elementos diferentes" (Morin, 2001, p.41). En un sentido similar, la idea de sistema se corresponde con un orden emergente, "cuyas características solo pueden ser inducidas una vez que el nuevo orden ya está constituido" (Rodríguez \& Torres, 2003, p. 113). De este modo, cuando se habla de sistema, el orden cualitativo emergente que nombramos puede deducirse a partir de las características de sus componentes; y para comprenderlo es preciso atender a la forma cómo se organizan entre sí los elementos que lo componen; a fin de que, posteriormente, se pueda explicar por qué y cómo interactúan a lo largo del tiempo: ¿se debe a factores externos o a una auto-organización y auto-reproducción?

Según los postulados teóricos de Luhmann 
(1998), un sistema autopoiético se individualiza en todos los casos en los que aparece la posibilidad de identificar un modo específico de operación, que se realiza al y solo al interior del sistema. Todos los sistemas autopoiéticos (entre los que están los individuos y las sociedades) se caracterizan por la clausura operativa. Este último concepto se refiere a que las operaciones que llevan a la producción de elementos nuevos de un sistema dependen de sus operaciones anteriores, y luego ellas se convierten en presupuesto para posibles operaciones ulteriores. Esto no significa que se trate de una repetición idéntica de lo mismo; la reproducción autopoiética debe entenderse como la creación constante de nuevos elementos vinculados a los precedentes y con posibilidades de ser conectados con los sucesivos debido a que todos son producidos por un mismo tipo de operación. "Autopoiesis significa, entonces sobre todo, determinación del estado siguiente del sistema a partir de la estructuración anterior a la que llegó la operación" (Rodríguez \& Torres, 2003, p.115).

¿Cómo se puede ejemplificar esto en materia de educación? Hay que imaginar a un profesor en un aula frente a su grupo de alumnos, participando en la sesión de un curso en cualquier escuela. Tal grupo, profesor, aula, horario y los tipos de relación que establecen, así como todo lo demás que les circunda, están rodeados de diferentes símbolos, costumbres, ritos, prácticas o formas; y en todo ello se sintetiza un tiempo sumamente largo de re-producción de las prácticas educativas a través de siglos. Además, estas últimas pueden pensarse como eventos que se producen en un momento preciso, pero desaparecen tan pronto como aparecen, haciendo un llamamiento a otros con los que se conectan y que deben suceder para permitir la continuidad $\mathrm{y}$, con ello, la constitución y reproducción del sistema (Arriaga, 2016).

Entonces, surge la pregunta: ¿por qué interactúan los elementos constituyentes del sistema a lo largo del tiempo? o, dicho de otro modo: ¿cómo y bajo qué mecanismos ocurren cada día nuevos eventos en las aulas de cada una de las instituciones educativas? Se puede sugerir que cada acto educativo (cada día, en cada aula, con cada grupo) pretende responder a la necesidad de enseñar que tiene toda sociedad, pero para ello toma como antecedente referencial a los que han tenido lugar antes; $y$, de la misma manera, lo que tiene lugar hoy en las aulas se convierte en el presupuesto para lo que se presume ocurrirá mañana. De esta manera, sin importar que al año siguiente cambien los alumnos, el profesor, el horario o las autoridades de la escuela, la clausura operativa del sistema constituye la base de su autonomía y permite, por una parte, distinguirlo de su entorno $\mathrm{y}$, por la otra, mantener su estructura; sobre todo si abstraemos el término "sistema" de sus connotaciones biológicas o cibernéticas y lo limitamos -como lo hace Luhmann- a la comprensión del comportamiento de los sistemas con un grado evolutivo mayor, como son los seres humanos y sus sociedades.

En este punto es preciso explicar por qué 
se puede entender a la educación como un sistema parcial de la sociedad contemporánea. Luhmann y Schorr (1993) proponen la siguiente tesis al respecto: "la sociedad moderna puede ser descrita como un sistema social que está estructurado primariamente sobre la base de una diferenciación funcional" (p.55). Esto significa que la sociedad contemporánea está constituida por sistemas parciales autopoiéticos y organizados en torno a una función específica. Los más claramente identificables serían el sistema político, el económico, el de la ciencia, el jurídico y, por supuesto, el de la educación. Una característica común en todos ellos -explican Luhmann y Schorr- es la separación de dos niveles de dirección del comportamiento: el de la codificación y el de la programación. El primero es un código binario, en tanto que el segundo reúne normas y criterios para una correcta adscripción de los valores del código (Luhmann \& Schorr, 1993).

En este sentido, el sistema Educación estaría codificado por la gestión que hace de "la carrera" del individuo calificando el buen/mal aprovechamiento de lo que debe aprender. En la hipótesis de Luhmann, el término "carrera" debe tomarse como el conjunto de sucesos que pueden cambiar la posición del individuo dentro de la sociedad; y se explica desde una sociedad en la que:

el individuo no se ve situado por su pertenencia a una determinada familia o a un cierto linaje; la individualidad ya no viene definida por la 'localización' (status, condición) (...)
El individuo es concebido como un sujeto que se determina a sí mismo de forma autónoma. Son los propios sistemas funcionales los que definen y condicionan lo que tiene valor para ellos, los que determinan y deciden la inclusión social del sujeto. Esta inclusión pierde con ello para el individuo el carácter de un status fijado por nacimiento y pasa a adquirir el carácter de carrera. (Luhmann \& Schorr, 1993, p.57)

En otras palabras, lo que hace el sistema Educación es codificar la selección social, pero lo hace en sus propios términos (criterios pedagógicos, didácticos, académicos), a través de sus planes curriculares y programación, que siempre se traducirán en el elogio o la censura, la aprobación o reprobación, el acceso a un nivel superior o la no admisión, el otorgamiento de un título o no, etcétera. "Todo proceso educativo es, por naturaleza, un proceso selectivo", dicen Luhmann y Schorr (1993, p.61). Y los criterios bueno/malo de su código binario establecen un patrón evaluativo de determinados rendimientos; no se trata de juicios morales o éticos que el docente realiza de cada estudiante, sino de la observación de normas para asignar cada uno de los valores del código.

Se advierte de inmediato una consecuencia de corte epistemológico, porque los sistemas sociales y los sistemas psíquicos comparten la característica de ser productores de sentido. Eso significa que la operación de ambos tipos de sistema (en forma de comunicaciones o de pensamientos) crea realidad fenoménica y, dado que 
el sentido delimita la observación del sistema, la diferenciación que resulta de aplicar el código correspondiente a cada sistema es estructurante del mismo y condiciona la realidad fenoménica que produzca. Dicho en otras palabras, la forma en que cada uno aprende a mirar el mundo es la forma en que se observará a sí mismo, pensará al mundo, actuará en él y, a su vez, el mundo actuará sobre cada individuo en forma similar.

Así, cuando alguien tiene el interés por investigar las reglas y códigos epistemológicos que recibe un estudiante mediante la educación formal, debe dar cuenta de la diferenciación basal del sistema que ha producido tales ideas o comunicaciones, debido a que esa diferenciación base condiciona la observación que el individuo puede hacer de su entorno. Esto solo puede hacerse -según la Teoría de Sistemascon una observación de segundo orden: la que observa al observador observando. Como se ve, la ascendencia constructivista que tiene la racionalidad sistémica es innegable; bien lo resumen Rodríguez y Torres (2003): en la propuesta teórica de Luhmann "el sujeto es remplazado por el observador y el objeto por lo observado", pero no se trata ya de un observador que limita su acción a la mera pasividad, al simple hecho de dejarse impresionar por el objeto observado. "El observador reconoce en esta teoría una posición más activa, conoce mediante esquemas de distinción que ha incorporado autorreferencialmente y que le permiten establecer diferencias recibiendo, por tanto, noticias de diferencia” (p.107).

De tal suerte, si se acepta como hipótesis que el sistema Educación es un sistema parcial autopoiético y cumple una función dentro de la sociedad, debe asumirse que las prácticas educativas concernientes se extinguirían en tanto dejen de cumplir la función que socialmente se les asigna, la cual es necesariamente instructiva y selectiva. La auto-reproducción del sistema depende de la recursividad de su operación específica, misma que se realiza con expectativas estandarizadas de habilidad y saber. Como hemos afirmado al comienzo, tales expectativas estandarizadas pueden pensarse como reducción de complejidad y anulación de la contingencia, lo cual pone a la labor educativa institucionalizada en calidad de proceso evaluable. A guisa de ejemplo está el Programme for International Student Assessment (PISA) de la OCDE (Organización para la Cooperación y el Desarrollo Económicos) que, en México, el Instituto Nacional para la Evaluación de la Educación (INEE) define como un instrumento que "mide si los estudiantes tienen la capacidad de reproducir lo que han aprendido" (INEE, 2016). Así queda claro que la asignación del valor positivo o negativo del código que aplica el sistema Educación se convierte en un principio a partir del cual se evalúa el correcto funcionamiento del sistema, pero a través del desempeño de los estudiantes.

Pero no solo una prueba estandarizada como PISA, sino la evaluación que permanentemente realizan los profesores en cada escuela, muestra que, más allá de los fines filosóficoeducativos de perseguir el ser humano ideal a 
partir de la educación, la evaluación del sistema busca aplicar el código ya descrito, mediante su programación, con lo que se termina tratando "al educando como si fuera una 'máquina trivial'. Las máquinas triviales se distinguen de las que no lo son (por ejemplo, las 'máquinas Turing') en que, merced a una regulación fijada como constante, reaccionan a un determinado input, produciendo un determinado output" (Luhmann \& Schorr, 1993, p.58).

Los output que el sistema Educación espera (y califica en los estudiantes, asignando el valor positivo o negativo del código) dentro de los sistemas de evaluación estandarizados buscan -dice el INEE- "conocer el nivel de habilidades necesarias que han adquirido los estudiantes para participar plenamente en la sociedad, centrándose en dominios claves como Lectura, Ciencias y Matemáticas" (INEE, 2016). Las máquinas triviales pueden ser valoradas bajo el principio de que conociendo todos los inputs, se pueden establecer todos los outputs. Es claro que los estudiantes son un tipo de sistema no trivial, pero parece que los mecanismos estandarizados de evaluación parten de una premisa según la cual si se tienen claro, por escrito y en forma programática, los contenidos de los cursos (input), es posible no solo predecir sino calificar los output.

Ya en otro trabajo (Arriaga, 2016), se explicaba que al interior de la clausura autopoiética del sistema Educación hay una serie de prácticas, costumbres, ideas y represen- taciones respecto a la acción educativa que no se formaron en esta época, en esta sociedad, ni en las actuales instituciones educativas, aunque hoy en día se re-creen constantemente a manera de eventos de temporalidad nula, a partir del modo específico de operación del sistema. El resultado, como dice Rogers (1996), es que se ha naturalizado la idea de que enseñar consiste en mantener el orden en la clase, comunicar hechos -por lo común mediante disertaciones y textos-, efectuar exámenes y adjudicar puntos.

A partir de los elementos expuestos hasta aquí (solo a manera de propuesta de perspectiva utilizando la metáfora del sistema), se puede decir que si la existencia del sistema Educación depende de su auto-reproducción, entonces la educación se hace todos días en las escuelas, en las aulas; y los individuos que concurren a tales espacios producen ideas (operación exclusiva de los sistemas psíquicos) que no solo orientan su comportamiento específico en el espacio escolar, sino que se comunican (lo cual es un principio básico en la Teoría de los Sistemas Sociales) con las de otros actores, como profesores, autoridades, compañeros, etc. y todo ello permite la recursividad del acto educativo que casi todo mundo idealiza en la siguiente fórmula: los estudiantes acuden a la escuela para aprender y son los profesores quienes les enseñan; si aprenden, aprobarán y continuarán su carrera. "Los padres de familia, el gobierno, las empresas y todo el mundo tienen tal expectativa; es más, es algo que han esperado de la educación durante siglos y esa expectativa no solo se renueva 
costeando nuevas escuelas, sino enviando a ellas a las nuevas generaciones" (Arriaga, 2016, pp. 132-133).

Hay que revisar dos cuestiones más para completar los desafíos cognitivos de la educación vista desde la perspectiva sistémica. La primera es que los resultados que se esperan de las prácticas educativas no apuntan al sistema social sino a su entorno: los sistemas psíquicos o individuos (por eso es que se evalúa el funcionamiento del sistema calificando los outputs de los estudiantes); la segunda consiste en que, del adecuado funcionamiento del sistema Educación, dependerá, en buena medida, la constitución y operación del sistema social, pues esos individuos interactúan todo el tiempo y consiguen auto-organizarse a niveles crecientes de complejidad, produciendo sistemas emergentes cuyos agentes parecen obedecer a reglas simples (Holland, 2000).

Se puede decir que sobre los resultados del sistema Educación visibles en los individuos seguramente no habrá gran discrepancia entre antropólogos, sociólogos, pedagogos y hasta filósofos en el hecho de que los estudiantes no deben verse como "máquinas triviales", pues no se puede decir que actúen como autómatas; no obstante, el sistema Educación termina tratándolos de ese modo, debido a que socialmente se le pide instruirlos, evaluarlos y aprobar a quien lo merezca. Así, lo que el estudiante recibe en las escuelas es complejidad reducida (input); en tanto que la aplicación de su código estructu- rante y sus normas (a través de la evaluación) remiten a la contingencia y a la necesidad de selección entre diversas alternativas: el estudiante que selecciona la respuesta adecuada en una evaluación recibirá el valor positivo del código binario que estructura el sistema, y ello le permitirá continuar su carrera para posicionarse socialmente.

Esta última idea (de recibir complejidad reducida) se puede ejemplificar así: cada persona tiene que decidir diariamente cosas como qué comer, de dónde obtener ese alimento, cómo prepararlo, con quién compartirlo, etcétera. Cada selección que realiza es contingente, así que tras cada elección de un individuo es posible identificar (en forma latente) el ser de otras posibilidades que no seleccionó. Dadas estas características, es fácil notar lo complicado y poco funcional de tomar decisiones distintas cada día y cada momento entre un mar de posibilidades, por lo que -según la metáfora sistémica- "un sistema se conforma de posibilidades que reduzcan las opciones a través de la conexión recursiva de las selecciones" (Ayala \& Arriaga, 2014, p.17). En tal sentido, lo que la educación formal hace es básicamente capacitar y socializar a los individuos, lo cual consistiría en reducir incertidumbre, proporcionar orden y sentido (cosa muy relevante si se toma en cuenta que se trata de "sistemas abiertos", en constante interacción con el entorno, que requieren controlar el acceso a posibilidades distintas a lo factual).

Dado que la teoría de los sistemas sociales 
de Luhmann "se originó en el pensamiento complejo de las ciencias físicas, desarrollado durante la década de los sesenta" (Carretero \& García, 2016, p.23), y debido a que la característica más importante de los sistemas autoorganizados y auto-referentes es la complejidad, es absolutamente pertinente su uso con fines heurísticos, sobre todo tratándose de los sistemas vivos que han alcanzado el mayor nivel de complejidad: los seres humanos y la sociedad.

En la siguiente parte del trabajo se estudiará la estructuración de las selecciones a partir de la operación específica del sistema Educación y de cómo ello organiza la clausura operativa de los individuos. La intención es ver a estos últimos como expresión extrema de complejidad que, sin embargo, al pasar por el sistema Educación, son tratados como "máquinas triviales" (aunque no lo son esencialmente, sino que, por el contrario, son capaces de incrementar su complejidad [Morin, 2005]), porque así se posibilita la operación de dicho sistema.

\section{Los sistemas psíquicos y el no equilibrio informacional}

En su Introducción al pensamiento complejo, Morin (2001) recuerda la importancia heurística que representó la noción de "sistema abierto" (originalmente termodinámica) para la comprensión de los sistemas vivos: se trata de "sistemas cuya existencia y estructura dependen de una alimentación exterior no solamente material-energética, sino también organizacional-funcional" (2001, p.43). Los estudiantes, esos sistemas psíquicos a los que se ha referido la primera parte de este texto, son sistemas abiertos y se sabe, como lo han estudiado gente como Spencer, Cottrell, White, Kroeber o Adams, entre muchos otros, que en tanto organismos biológicos deben obtener inevitablemente energía de su entorno para sobrevivir. Este hecho puede ser puesto como primera evidencia de que se trata de sistemas abiertos, alejados del equilibrio termodinámico, capaces de evolucionar a formas cada vez más complejas de subsistencia. Pero para este trabajo interesa de manera particular hablar no solo de los flujos de masa y energía que los seres humanos intercambian con su entorno todo el tiempo, sino de las interacciones que mantienen permanentemente con este, obteniendo de él un orden informacional y un tipo de estructura que les permite auto-eco-organizarse (Ciurana \& Regalado, 2016).

Debe entenderse que los seres humanos son "máquinas no triviales" en el sentido de que su organización como sistema (sistema psíquico, cuya operación específica es la producción de ideas) se debe a sus interacciones con el entorno, aunque están dotados de autonomía. Morin (2001) lo explica de la siguiente manera:

el orden de lo viviente no es simple, no depende de la lógica que aplicamos a todas las cosas mecánicas, sino que postula una lógica de la complejidad (...) la individualidad del sistema viviente se distingue de aquella de otros sistemas cibernéticos (...) está dotado de autonomía, autonomía ciertamente 
relativa, debemos recordarlo ( $\sin$ cesar), pero autonomía organizacional, organísmica y existencial (...) Pero al mismo tiempo que el sistema auto-organizador se desprende del ambiente y se distingue de él, y de allí su autonomía e individualidad, se liga tanto más a ese ambiente al incrementar la apertura y el intercambio que acompañan a todo progreso de la complejidad. (pp.56-57)

De este planteamiento de Morin se debe destacar que el sistema psíquico obtiene información del ambiente, la cual termina por convertirse en orden. Para entender esto de manera adecuada, hay que clarificar este concepto. Como dice Gell-Mann, la información “tiene que ver con una selección entre diversas alternativas y puede expresarse de modo muy simple si dichas alternativas pueden reducirse a una serie de elecciones entre alternativas binarias igualmente probables" (2007, p.53). Como puede verse, no se recibe información en forma de insumo definido, sino como probabilidades $u$ opciones que deben someterse a elección. Debe decirse entonces que todo sistema (psíquico o social) orienta su observación y autopoiesis con base en una distinción, en selecciones recursivas que le ayudan a procesar la complejidad de su entorno, al que siempre observará según distinciones particulares propias.

Un ejemplo es el siguiente: en el entorno de un individuo $\mathrm{X}$ hay organizaciones, animales, otras personas, máquinas, etcétera; todos los cuales constituyen también sistemas y están diferenciados de sus respectivos entornos. Esta última diferenciación no depende del individuo $\mathrm{X}$; sin embargo, cuando este último dirige su mirada a esos sistemas y los piensa, termina por clasificarlos con base en la distinción que es estructurante de sí mismo, así, al mirarlos y pensarlos, puede considerarlos normales o anormales, útiles o inútiles, propios o extraños, etcétera. De tal modo que esa información (generada de la diferenciación aplicada a la observación del entorno) se convierte en el orden que el individuo $\mathrm{X}$ dará a su mundo. Por eso se dice que la información termina por convertirse en orden y también puede hablarse de que el humano es una máquina no trivial; mas bien es una máquina como la de Turing, que una vez que ha computado (computación en el sentido de computare en latín: considerar en conjunto) deja de ser la misma, ya ha cambiado.

Con lo anterior aparece el tema de la "epistemología de los sistemas observadores" (Von Foerster, 1998, p.638); concepción constructuvista que cambia radicalmente las cosas, porque con ella se deja de pensar en que las propiedades residen esencialmente en las cosas para sugerir, por el contrario, que radican en lo que emerge al aplicar los esquemas de distinción que estructuran la mirada del sistema psíquico. Es en el plano de las ideas donde primariamente los sistemas psíquicos aplican esos esquemas de distinción; pero, en tanto se trata de realidad fenoménica, también está implicada la acción en esto, pues las selecciones que se realizan cotidianamente (entre varias alternativas con 
iguales probabilidades de ser) se convierten en complejidad reducida, que luego debe encontrar la manera de comunicarse con otros sistemas psíquicos y -asombrosamente- lograr la coordinación que da vida a los sistemas sociales.

Ciertamente, educar es quizá la práctica más humana y es "muy anterior al pensamiento pedagógico. 'Aprender a' es una noción que se nos aparece desde la comunidad primitiva: para aprender a usar el arco, el niño cazaba, para aprender a nadar, nadaba" (Gadotti, 2008, p.7). Pero, si bien en toda sociedad humana están presentes procesos que facilitan el aprendizaje como medio que permite la continuidad (biológica, económica, simbólica, etc.), hay diferencias en las formas de hacerlo, entre, por ejemplo, las sociedades no alfabéticas respecto de las alfabéticas. También hay diferencia entre las "sociedades con escuelas" y aquellas en que "la instrucción no se da en un entorno físico dedicado a este fin, llevada a cabo por un maestro cuyo único papel es enseñar" (Le Vine, en Barfield, 2000, p.182).

En las sociedades con escuelas no solo se presenta una enseñanza basada en la lectura, sino que se conjugan las condiciones para tomar conciencia de la importancia de enseñar. La forma de pensar la educación en este tipo de sociedades ofrece la figura pedagógica por excelencia: el maestro. Verbigracia: según el testimonio de Diodoro Sículo del siglo I d.C. “...Entre otras cosas se dice que en Babilonia los caldeos eran antiguos colonos egipcios, expertos en astrología, por haberla aprendido de los sacerdotes egipcios" (Citado por Alighiero, 2007, p.16). Y, entonces, la fórmula "por-haberla-aprendido-de" revela la figura pedagógica del maestro: alguienque-enseña-algo-a-alguien. Si esa es la forma en que lo quiso decir Diodoro Sículo, emerge una manera específica de enseñar/aprender que es milenaria; pero incluso en el caso de que se trate de una cuestión atribuible al cómo fue traducido, este autor griego confirma en la contemporaneidad que en la época actual quien traduce piensa "naturalmente" en que es así como opera la enseñanza/aprendizaje: alguien-que-enseñaalgo-a-alguien. $Y$ esto significa transmisión de complejidad reducida.

No se puede afirmar de ninguna manera que en las "sociedades con escuelas" no se presente enseñanza/aprendizaje de otro tipo. La diferencia solo se plantea para subrayar la mayor organización y sistematización históricamente gestadas en este tipo de enseñanza/aprendizaje y que, mediante su recursividad, sientan las bases de procedimientos que hasta la fecha permanecen vigentes en varios niveles escolares. Por esa permanencia resulta casi automático para todos pensar a la educación y las escuelas con base en una imagen del aula, con un grupo de aprendices sentados uno al lado del otro, un maestro dirigiendo lo que se debe hacer, cómo se debe hacer y calificando el desempeño. Se puede intuir, incluso, que los cuerpos mismos van adquiriendo el habitus que se repetirá, una y otra vez, en quienes han pasado por esa situación durante siglos y siglos.

Ahora bien, la imagen mental que surge al 
utilizar la metáfora de los sistemas abiertos remite a un todo organizado, emergente, capaz de diferenciarse de su entorno, con una operación específica que lo sostiene de manera permanente en el tiempo, pero que depende de flujos externos. Si se le emplea para pensar en los estudiantes, se puede decir que su estabilidad es muy frágil y efímera, pues estos dependen de una alimentación exterior, no solamente materialenergética, sino también organizacional-informacional. Hay, por tanto, un desequilibrio en el flujo que los alimenta, pero ese desequilibrio es el que permite al sistema mantenerse en aparente equilibrio, es decir en estado de estabilidad y de continuidad; y ese equilibrio aparente no puede sino degradarse cuando queda librado a sí mismo, es decir, si hay clausura del sistema (Morin, 2001).

Esta última afirmación es la que debe subrayarse porque en ella reside la propuesta de apartarse de las condiciones que podrían derivar en una degradación del sistema (que en términos energéticos conduce a la entropía y el desorden, pero en términos informacionales puede llevar al dogmatismo, fundamentalismo y la no comprensión de la diversidad, que deriven en confrontaciones). Esta imagen del sistema abierto suele ilustrarse con la llama de una vela o con un tornado: en la medida que reciben del exterior flujos permanentes de energía o materia pueden seguir operando, pero cuando ello deja de ocurrir se extinguen. Igualmente, se puede hacer la comparación con un ser vivo, que alcanza un aparente equilibrio nutricional a partir de un flujo regular de nutrientes desde el exterior. Si ese flujo se detuviera, su vida correría peligro, pero desde el momento en que alcanza estabilidad se le puede pensar como una estructura. Es un error no darse cuenta que los componentes cambian como condición indispensable para que la estructura se mantenga; $y$ esto lleva a pensar en que el organismo lo que hace es organizar su clausura por estar abierto al exterior. Entonces, un abordaje más completo no solo sería para estudiar el sistema mismo sino también su relación con el ambiente porque es constitutiva de él.

Ahora, si como dice Gell-Mann (2007), los humanos son los organismos más complejos en la historia de la tierra, los flujos de que dependen no son solo energía y materia, sino también información y organización. El ser humano no puede vivir en la incertidumbre, en la falta de referentes, de sentido, de criterios para tomar decisiones; necesita imperiosa y vitalmente información proveniente del entorno. Como dicen Ciurana y Regalado, "si hay un estado mental que molesta es el estado de incertidumbre" (2016, p.16). Por esta razón, también se puede sugerir que, "desde que el hombre existe como tal sobre la tierra, ha dedicado gran parte de sus esfuerzos intelectuales a establecer cómo debe ser él. Esto lo ha llevado a regir su existencia de acuerdo a un campo de regularidad, separando lo 'normal' de lo 'anormal', como forma de afirmar su subjetividad" (Arriaga, 2002, p.94).

Pero también, si como propone Morin (2005), el hombre es un ser bio-psico-social, entonces 
requiere flujos provenientes del entorno que alimenten cada una de esas dimensiones. La universalidad de esas dimensiones para toda la raza humana no impide que combinaciones muy particulares de cada una de ellas den vida a expresiones diferenciadas del fenómeno humano, a partir de muy singulares sistemas de representaciones, creencias, organización política, bagaje cultural e ideológico de los grupos sociales (Guber, 2005). De hecho esta es la base epistemológica de la antropología: el fenómeno humano y sus múltiples expresiones.

Si se advierte, entonces, la diversidad del fenómeno humano, es claro que las dimensiones universales son alimentadas por los entornos de cada grupo humano, dando vida a versiones particulares de esas dimensiones universales. Los procesos que al interior de cada grupo se ponen en marcha para dar continuidad a sus respectivos sistemas culturales, sociales, políticos, económicos y de toda índole pasan por la transmisión de saberes. Justo aquí aparece esa fórmula esencial para la figura del maestro que se mencionó párrafos atrás: alguien-queenseña-algo-a-alguien. Esta fórmula no puede sino operar a partir de la transmisión de una complejidad reducida que precisamente corresponde al tipo de sociedad en la que se encuentre quien aprenderá la particular combinación de las dimensiones universales. Los contenidos que pueda aprender, sobre todo, a través de la educación formal, deben ser programáticos, con objetivos específicos, con técnica didáctica, evaluables. Todo ello remite a un problema epistemológico fundamental que ya se esbozó en el apartado anterior: la forma en que cada estudiante aprenda a mirar el mundo es la forma en que se observará a sí mismo, pensará al mundo, actuará en él y se dejará influenciar.

En resumen, los flujos de intercambio sistema/entorno en el caso de los seres humanos observan patrones ampliamente diversificados que se manifiestan en procesos adaptativos variopintos, haciendo posible la diversidad del fenómeno humano. Entonces, para la continuidad de un sistema tan complejo y adaptable como lo son los seres humanos, lo primero que se requeriría es asegurar un estado (que es constante y por lo tanto frágil), que se lograría mediante la clausura operativa que permita la autopoiesis. Alcanzar tal condición tiene algo de paradójico: las estructuras se mantienen mientras los constituyentes cambian. Esto no significa otra cosa que la siguiente "ley": el sistema debe cerrarse al mundo exterior a fin de mantener sus estructuras y su medio interno que, si no, se desintegrarían, pero es su apertura lo que permite su clausura (Morin, 2005, p.44).

$\mathrm{Si}$ es la apertura (representada en flujos energéticos o informacionales) lo que permite la clausura, parece aclararse una cuestión nodal: los sistemas psíquicos auto-organizan su operación específica (o sea la producción de pensamiento) con la información que reciben del exterior. Como se dijo antes, esto se puede expresar como un proceso que consiste en aprender a pensar a partir de la relación con el entorno. Como a este 
trabajo interesa específicamente el tema de la educación formal, es necesario asumir que en las escuelas se enseña a pensar y eso significa que se transmiten las instrucciones para la clausura operativa del sistema psíquico que se estructura, por un lado, a partir del código binario al cual se aludió en el primer apartado; y luego se traduce en programas para asegurarse de que el sistema (psíquico) reciba los inputs adecuados, con lo cual se buscan outputs específicos (evaluables). Los contenidos temáticos de cada curso que se imparte en las escuelas implican una selección (hecha con criterios disciplinarios, pedagógicos, jurídicos o lo que apliquen) y el objetivo de transmitirlos y evaluar su asimilación no es sino la programación del sistema. Esa es una característica inmanente del sistema.

Conforme cada individuo va siendo incluido en el sistema de Educación (y sometido a la asignación de un lugar dentro o fuera de él con la aplicación del código mejor/peor aprovechamiento), "aunque sin tener plena conciencia de ello, (elabora) un conjunto provisional de reglas" (Gell-Mann, 2007, p.69) y se involucra en un mejoramiento progresivo de ese conjunto, de modo que va formando una gramática de su "carrera", es decir, una manera de organizar y combinar elementos, en otras palabras, una manera de pensar. Justo ahí es donde va alcanzando esa clausura operativa que requiere como sistema psíquico para operar y garantizar su continuidad.

Retomando a Luhmann y Schorr, el sistema Educación gestiona una porción de la "carrera" del individuo, pero este último (en tanto sistema que depende de los intercambios con el exterior) tiene la capacidad de comprimir ciertas regularidades (mismas que identifica con base en su cúmulo de experiencias al interior del sistema Educación) en un esquema compuesto de reglas que gobiernan sus experiencias, pero que en realidad omiten las circunstancias especiales en las que deben aplicarse (en la escuela). De ese modo, el individuo obtiene una condensación de sentido o expectativa que ordena lo contingente en el sentido amplio, o sea, organiza su clausura autopoiética. Así es como el pensamiento del estudiante se estructura y llega a poseer un esquema de distinción que le permite seguir observando y conociendo.

Para finalizar este apartado y dar paso al último, en el que se esboza una propuesta para evitar que la clausura operativa lleve a la degradación del sistema, debe subrayarse que las leyes de organización de lo viviente son de desequilibrio, de dinamismo estabilizado; y la estabilidad del sistema debe encontrarse no solamente en el sistema mismo, sino también en su relación con el ambiente, sin embargo, esa relación no constituye una simple dependencia, sino que más bien es constitutiva del sistema.

\section{La clausura autopoiética y el riesgo de} degradación del sistema

De acuerdo con la perspectiva que se desarrolló en este trabajo, las prácticas educativas en la sociedad contemporánea cumplen la función de instruir y asignar o negar a cada 
individuo un lugar dentro del sistema social, y lo hacen a partir de su contribución a la clausura operativa del sistema psíquico (productor de pensamientos). El orden transmitido a través de los flujos de información consigue la estabilidad de ese sistema, básicamente recurriendo a una fórmula que se pone a prueba en este apartado: cuando se está seguro de algo es posible llegar a un estado en el que no hace falta buscar más respuestas $\mathrm{y}$, a la vez, se alcanza un cierto equilibrio/orden informacional que permite la generación de los nuevos elementos (ideas o pensamientos) que produzca el sistema.

Descrito de esa manera parece algo simple, pero Morin sugiere que "lo simple no es más que un pasaje, un momento entre dos complejidades, la microfísica y la macro-cosmo-física" (Morin, 1981, p.41). En este sentido, se puede hacer el siguiente planteamiento con base en la metáfora de la entropía: dado que este término fue acuñado por la termodinámica para ofrecer la imagen de un sistema que llega a desorganizarse, como premisa nos lleva a pensar en la conversión energética que realiza un sistema; y lanza el desafío de preguntarse por qué inexorablemente deviene en entropía. Como dice Zaid (2013), "la degradación (de energía) avanza con el tiempo de modo irreversible: implica un antes y un después venido a menos (...) La energía, el calor, el movimiento tienden a disiparse, enfriarse, nivelarse, detenerse. La vida tiende a desorganizarse, la comunicación tiende al ruido, el orden al desorden. Todo tiende a menos: a la degradación que se llama entropía” (s/p).

Por otra parte, como se ha planteado en este trabajo, el concepto de información es inseparable del de organización. Se emplearon las metáforas de sistema y de autopoiesis para imaginar a los individuos que forman parte del sistema Educación, organizando su propia clausura operativa, en tanto sistemas psíquicos, a partir del intercambio de información con el entorno del que dependen. Así, en las aulas, los estudiantes intercambian constantemente información y consecuentemente organización para cada uno de ellos; y normalmente lo hacen a partir de complejidad reducida. De esta manera, el individuo alcanza un equilibrio (precario, frágil, inestable), que no le exenta de devenir en desorden y degradación del modo que lo sugiere el principio de la entropía, debido a la estabilidad que se alcanza (que reduce incertidumbre) y que incuso es estimulada con evaluaciones positivas: elegir las respuestas correctas, no equivocarse para no ser reprobado.

Se podría afirmar que la información es el incremento de la organización, o sea de la complejidad (Morin, 2005). Esto equivaldría a decir que mientras más información fluya hacia el sistema psíquico, este verá incrementar el volumen de selecciones, pero eso depende de cómo se gestionen esos estímulos, bajo qué esquemas de diferenciación sean observados. Si los esquemas son disyuntivos y reduccionistas, el riesgo de que el sistema se degrade es alto. La degradación deviene entonces por no saber cómo gestionar el hecho de que el sistema es autónomo pero no puede estar aislado: necesita materia/energía e información. Por eso Morin (2001) sostiene que el ambiente está dentro 
del sistema (y este lo que hace es organizar su clausura), jugando un rol de co-organizador. Un sistema auto-eco-organizador no puede bastarse a sí mismo, únicamente se puede volver lógico cuando introduce al ambiente en él por la vía de la información. Sin embargo, cuando ese ambiente está lleno de otros sistemas que parecen caóticos (por ser distintos, por obedecer a otras lógicas, a diferentes formas de reducción de complejidad, a otras unidades que aglutinan combinaciones muy particulares de las dimensiones universales de lo humano), el problema se vuelve de convivencia.

En este sentido, hay un desafío abierto que se ha reconocido como problema paradigmático de la sociedad actual: ¿podemos seguir viviendo juntos, iguales y diferentes? (Touraine, 2001). Y la respuesta discursiva que habla de la interculturalidad, la tolerancia, la solidaridad, la inclusión, etcétera, corre el riesgo de convertirse en un universalismo abstracto (Morin, 2001) por carecer del principio epistemológico que surja tras sentir la necesidad de comprender la multiplicidad, de entender al otro y de reconocerle como interlocutor que le complementa, precisamente por su "no ser como yo".

Si se piensa al sistema psíquico y su clausura operativa organizada en las aulas (bajo un esquema binario), parecería que una condicionante para afrontar la multiplicidad contemporánea es el posicionamiento frente a la fragmentación, la desarticulación y el anatema ante lo diferente. En otros tiempos, los encuentros con la otredad solían ser propiciados por la gente con intereses antropológicos, en un afán por ir al encuentro con lo distinto y documentar las diversas expresiones del fenómeno humano. Pero, en una época como la actual, los intercambios comerciales globales, la acelerada urbanización, los movimientos migratorios, el flujo masivo de datos en tiempo real, la proximidad acortada por las vías de comunicación, entre otros procesos, han "forzado" la convivencia entre grupos humanos diversos.

La experimentación de esa proximidad intercultural puede devenir en al menos dos posibilidades: la primera, una degradación del sistema debido al incremento de información pero organizada a partir de una diferenciación basal dicotómica, reduccionista, fragmentadora. Tal degradación vendría de la incapacidad de gestionar más complejidad (Ciurana \& Regalado, 2016). La metáfora de la entropía serviría en este caso para decir que es "como si" el flujo energético que requiere un molino de viento para seguir girando no le viniera solo de este a oeste, sino que otras rachas llegaran -al mismo tiempo- de sur a norte, de oeste a este $\mathrm{y}$ de norte a sur. Cuando un individuo se ve a sí mismo viviendo en un mundo con cambios tan acelerados, con distancias acortadas, con movimientos globales, con movimientos locales, con identidades emergentes y múltiples, etcétera, corre el riesgo de devenir en degradación por carecer de los medios para procesar esa información y traducirla en orden a su interior.

La otra posibilidad consiste en adoptar una epistemología abocada a la comprensión de los 
fenómenos no reductibles a una sola dimensión (Ciurana \& Regalado, 2016), y que más bien muestre interés por las características emergentes, propias del devenir, la indeterminación y la contingencia. Los fenómenos emergentes del mundo contemporáneo difícilmente se pueden explicar desde el determinismo y la causalidad; es necesario hacerlo con categorías como desorden, novedad, incertidumbre, evolución, adaptación, complejidad creciente, entre otras. Y eso implica re-organizar la clausura autopoiética, pero ahora bajo un nuevo tipo de diferenciación, más relacional y menos reduccionista. Se requeriría una diferenciación de tipo dialógico, que no anula lo que no cabe en la diferencia, sino que logra un acoplamiento que genera evolución, que "complejiza el pensamiento y configura sujetos críticos, innovadores, responsables y reflexivos; capaces de repensar la realidad, valorar el trabajo y la sociedad, así como de tomar decisiones en consideración a la naturaleza humana y social" (Ruedas, 2016, p.38).

\section{Conclusiones}

Al principio del texto se expresaba la paradoja que encierra el tema educación y complejidad. Tras lo planteado en los tres apartados del trabajo, hay algunas cosas que parecen más claras: primero, que si bien la educación formal procede a partir de la selección de contenidos $\mathrm{y}$ su impartición se hace con base en la asignación de los valores mejor/peor aprovechamiento, ello es necesario para organizar la clausura operativa de los sistemas psíquicos; $y$, sin embargo, no es suficiente. Un buen número de los problemas de convivencia actuales (intolerancia, xenofobia, racismo, exclusión, etcétera) tienen que ver con la conservación del orden que se generó en esa "primaria" clausura operativa. En estas circunstancias, el sujeto debe afrontar el problema de incrementar la complejidad de su operación específica (producir ideas-acciones), para permitir que lo que ocurre en su entorno pueda seguir alimentando el orden informacional en el que se desarrolla. Los estudiantes no son máquinas triviales, tienen la capacidad de actuar de modos nuevos en momentos de crisis; por eso, ante la discrepancia entre el orden primario que ayudó a organizar la clausura operativa y un entorno cambiante, dinámico, con mucha mayor información que incorporar, se abre la posibilidad de superar la paradoja aumentando complejidad intelectual. Es un reto del presente que también es un desafío a la convivencia: mirar las dimensiones universales que tienen en común las distintas expresiones del ser humano y que pueden ser la base de la comunicación, el diálogo y la gestión de soluciones.

\section{Referencias}

Alighiero, M. M.(2007). Historia de la Educación 1 de la antigüedad al 1500. México: Siglo XXI editores.

Arriaga, J. L. (2002). En la búsqueda del ser humano universal. Derechos Humanos y discapacidad. En Derechos Humanos de las personas con discapacidad. México: CNDH.

Arriaga, J. L. (2016). Contingencia y mentalidad en la innovación curricular., Buenos Aires: Dunken. 
Ayala, G. \& Arriaga, J. (2014). Investigar la migración en tanto factor que ejerce presión sobre los sistemas sociales: propuesta metodológica a partir de Luhmann. Paradigmas, 6(1), 11-35.

Carretero, R. \& García, C. (2016). NiklasLuhmann, inclusión y diferenciación teórica. Metapolítica, 20(92), 22-25.

Ciurana, E. \& Regalado, C. (2016). Pensamiento complejo y educación. Aclaraciones $y$ confrontaciones. México: Frontera Abierta.

Contreras, J. (1994). Enseñanza, curriculum y profesorado. Madrid: Akal.

Corsi, G., Esposito, E., \& Baraldi, C. (2006). Glosario sobre la teoría social de Niklas Luhmann. México: Universidad Iberoamericana.

Gadotti, M. (2008). Historia de las ideas pedagógicas. México: Siglo XXI.

Gell-Mann, M. (2007). El Quark y el jaguar. Aventuras en lo simple y lo complejo. Barcelona: Tusquets.

Guber, R. (2005). El salvaje metropolitano. Buenos Aires: Paidós.

Holland, J. (2000). Emergence: From Chaos to Order. New York: Oxford University Press.

Instituto Nacional para la Evaluación de la Educación (2016). PISA ¿Preguntas frecuentes? Recuperado desde: http:// www.inee.edu.mx/index.php/proyectos/ pisa/que-es-pisa.

Le Vine, R. (2000). Educación. En T. Barfield, (editor), Diccionario de Antropología. México: Siglo XXI.

Luhmann, N. \& Schorr, K. (1993). El Sistema
Educativo (Problemas de Reflexión). México: Universidad de Guadalajara, Universidad Iberoamericana e ITESO.

Luhmann, N. (1998). Teoría de los Sistemas Sociales (Artículos). México: Universidad Iberoamericana.

Mascareño, A. (2016). La incompletitud de la autopoiesis. Irritación, codificación y crisis. Metapolítica, 20(92), 26-35.

Maturana, H. \& Varela, F. (2003). De máquinas y seres vivos. Autopoiesis: la organización de lo vivo. Buenos Aires: Lumen.

Morin, E. (1981). El Método I. Madrid: Cátedra. Morin, E. (2001). Introducción al pensamiento complejo. Barcelona: Gedisa.

Morin, E. (2005). El paradigma perdido. Ensayo de bioantropología. Barcelona: Kairós.

Najmanovich, D. (2008). Mirar con nuevos ojos. Buenos Aires: Cátedra.

Rodríguez, D. \& Torres, J. (2003). Autopoiesis, la unidad de una diferencia: Luhmann y Maturana. Sociológicas, 5(9), 106-140.

Rogers, C. (1996). Libertad y creatividad en la educación. Barcelona: Paidós Ibérica.

Ruedas, M. (2016). Aportes axiológicos de experiencias didácticas complejizantes en la formación de docentes. Educación y humanismo, 18(30), 28-41.

Touraine, A. (2001). ¿Podremos vivir juntos? México: F.C.E.

Von Foerster, H. (1998). Por una nueva epistemología. Metapolítica, 2(8), 629-641.

Zaid, G. (2013). Metabolismo del progreso. Letras libres, 177. Recuperado desde: http://www.letraslibres.com/hemeroteca/ edicion-mexico/estados-unidos-el-amorpor-las- armas. 